\title{
Búsqueda de sensaciones, autoconcepto, asertividad y consumo de drogas ¿Existe relación?
}

\author{
Manuela Martínez-Lorca*, Carlos Alonso-Sanz** \\ * Departamento de Psicología. Universidad de Castilla-La Mancha. Profesora Asociada. \\ ** Servicio de Drogodependencias. Junta de Comunidades de Castilla-La Mancha. \\ Enviar correspondencia: \\ Manuela Martínez-Lorca. CENTRO DE ESTUDIOS UNIVERSITARIOS. Avd. de la Real Fábrica de las Sedas, s.n. \\ 45600 Talavera de la Reina (Toledo).Tno.: 925 721010. mmlorca72@hotmail.com
}

\section{RESUMEN}

Este trabajo tiene como objetivo analizar si existe relación entre determinadas variables de personalidad (búsqueda de sensaciones, autoconcepto y asertividad) y el consumo de tabaco, alcohol y cannabis.

La muestra está formada por 243 sujetos de $1^{\circ}$ ESO correspondientes a 4 colegios de Toledo. Los resultados ponen de manifiesto la intensa relación que tiene la búsqueda de sensaciones con el consumo de drogas. En cuanto al autoconcepto indican que, aunque el autoconcepto general tiene poca relación con esta conducta, el autoconcepto familiar y académico sí parecen tener un efecto protector respecto al consumo de drogas. Ello subraya la importancia preventiva del ámbito familiar y la necesidad de trabajar sobre la mejora del autoconcepto académico, más que sobre el puro rendimiento escolar, ya que este último ha mostrado escasa relación con el consumo de drogas. En cuanto al autoconcepto social tiene también poca relación con el consumo de sustancias, lo cual parece desaconsejar que los programas de prevención se centren en el desarrollo de habilidades sociales generales, y es consistente con la débil relación encontrada entre la asertividad y el consumo de drogas. El autoconcepto emocional, en contra de lo esperado, muestra una relación directa con el consumo de las sustancias estudiadas.

Palabras clave: Búsqueda de sensaciones, autoconcepto, asertividad, población escolar, consumo de drogas.

\section{ABSTRACT}

The purpose of the present work is to analyse if there is a relationship between certain personality variables (sensationseeking, self-concept and assertiveness) and tobacco, alcohol and cannabis use.

The sample consisted of 243, 12 year old students of 4 schools in Toledo. The results indicated the strong relationship between sensation-seeking and drug use. As far as the selfconcept, the results indicated that, although general self-concept has little relation with behaviour, the family and academic self-concept do seem to have a protector effect in drug-use. This underlines the preventive importance of the family environment and the necessity of working to improve academic self-concept, rather than the purely academic performance, as the latter has shown little relation with drug-use. As far as the social-concept is concerned, there is also little relation with substances use, which seems to advise against basing prevention programmes on the development of general social skills, and is consistent with the weak relationship found between assertiveness and drug-use. Emotional self-concept, contrary to what we expected, indicates a direct relation with the use of the substances studied.

Key words: sensation seeking, self-concept, assertiveness, school students, drug use.

\section{INTRODUCCIÓN}

I consumo de drogas, especialmente de sustancias legales, constituye uno de los problemas más importantes con los que se enfrenta la sociedad actual ya que ocasiona graves costes personales, sociales y económicos. Además, esta conducta es más preocupante cuando se produce en población infantil y juvenil, no sólo por los problemas inmediatos que puede generar, sino también por la consolidación del consumo que conlleva su inicio en edades tempranas.

Recientes estudios (Observatorio Español sobre Drogas, 2000; Sáiz, González, Jiménez et al. 1999; Graña, Muñoz-Rivas, Andreu et al. 2000; Espada, Méndez e Hidalgo, 2000) señalan que existe un porcentaje relativamente alto de escolares que ha tenido contacto con las distintas drogas, sobre todo con alcohol, tabaco y cannabis. 
Numerosos trabajos han investigado la influencia de distintos factores de riesgo vinculados a la aparición del consumo de drogas, destacando por su relevancia la relación existente entre algunas variables de personalidad y dicho consumo.

Por ejemplo, González, Ibáñez y Peñate (1997) encontraron relaciones del consumo frecuente de alcohol con psicoticismo y extraversión. Estos mismos autores también llegaron a la conclusión de que la búsqueda de sensaciones, y más concretamente el factor desinhibición, están estrechamente relacionados con el consumo de alcohol. En el mismo sentido, Luengo, Otero-López, Romero et al. (1996) encontraron en población escolar que el factor de búsqueda de sensaciones más relacionado con el consumo de drogas legales es la desinhibición y el más relacionado con el consumo de cannabis es la búsqueda de experiencias.

Igualmente Sáiz, González, Jiménez et al. (1999) han encontrado que el consumo de drogas se asocia con mayores niveles de inestabilidad emocional, extraversión y psicoticismo, así como con un marcado perfil de búsqueda de sensaciones.

Datos similares se encuentran para el consumo de MDMA en población escolar española, obteniéndose puntuaciones más elevadas en las escalas de psicoticismo y búsqueda de sensaciones entre los jóvenes que han usado alguna vez esta sustancia (Sáiz, González, Paredes et al, 2001).

Otra variable ampliamente relacionada con el consumo de drogas es el autoconcepto. Así, Romero, Luengo y Otero-López (1995) concluyen que tener una baja autoestima familiar y escolar y una alta autoestima en el grupo de iguales está asociado al consumo de drogas en población escolar. Igualmente, Jackson (1997) encuentra que los escolares con alta autoestima están menos implicados en la iniciación y experimentación hacia el consumo de tabaco y alcohol.

También en población escolar, Graña y MuñozRivas (2000) confirmaron que los principales factores de riesgo psicológicos para explicar el consumo de drogas legales eran la autoestima, la presencia de conductas antisociales y la desinhibición, siendo los factores de protección más importantes el concepto positivo de uno mismo, el nivel de sinceridad y la práctica religiosa.

También Graña, Muñoz-Rivas, Andreu et al. (2000) encuentran que los jóvenes que habitualmente beben, fuman y consumen cannabis, mantienen un bajo concepto de sí mismos.

En cuanto a la asertividad, Rhodes y Jason (1990) concluyen que entre los factores que influyen en el consumo de drogas hay que destacar la pobreza familiar y la baja asertividad. Sin embargo Ashby, Baker y Botvin (1989) sostienen que la asertividad general no está relacionada con el consumo de tabaco, alcohol y marihuana, aunque sí lo están la asertividad social y la asertividad relacionada con sustancias, de forma que los sujetos con más riesgo de consumir drogas son los que puntúan alto en asertividad social y bajo en asertividad relacionada con las sustancias. También sobre este tema, Gustafson y Kälmén (1996) encontraron que los sujetos con alta asertividad, después de consumir alcohol, presentan una menor asertividad, mientras que los sujetos con baja asertividad, después de beber alcohol, tienen mayor asertividad. Por ello la intoxicación alcohólica produce cambios en la asertividad, desinhibiendo a los sujetos con baja asertividad e inhibiendo a los de alta asertividad.

Considerando la relevancia de estas variables en el inicio del consumo de drogas y en el diseño de programas de prevención, el objetivo del presente trabajo es profundizar en el estudio de la relación entre búsqueda de sensaciones, autoconcepto y asertividad con el consumo de drogas legales e ilegales (tabaco, alcohol y cannabis), y también estudiar las relaciones existentes entre estos factores de personalidad y un amplio conjunto de variables asociadas al consumo de dichas sustancias.

\section{MÉTODO}

\subsection{Instrumentos utilizados}

Para la recogida de datos se utilizó un cuestionario elaborado "ad hoc", el cual incluye ítems referentes a las características sociodemográficas de los sujetos y a las siguientes variables relacionadas con el consumo de tabaco, alcohol y cannabis: cantidad de oferta recibida, consumo en vida, consumo actual (últimos 30 días y semana pasada), intención de consumir en el futuro, normas subjetivas (rechazo percibido a su consumo de drogas en padre, madre y mejor amigo/a), consumo del mejor amigo/a, conocimientos sobre las drogas, actitudes hacia las drogas, consumo abusivo de alcohol ( $n^{\circ}$ total de episodios de embriaguez y en los últimos 30 días), expectativas negativas y positivas respecto al consumo, prevalencia percibida, probabilidad de rechazar ofertas de drogas, imagen de consumidor, consumo de drogas en presencia de los padres, dificultad percibida para obtener drogas, rendimiento escolar y consumo familiar.

Además se aplicaron tres cuestionarios para la medida de las variables de personalidad:

La búsqueda de sensaciones se midió mediante la Escala de Búsqueda de Sensaciones para Niños y Adolescentes (EBS-J, Pérez, Ortet, Plá et al., 1987) derivada de la "Escala de Búsqueda de Sensaciones" (Sensation Seeking Scale, SSS, Zuckerman, Eysenck y Eysenck, 1978). Consta de 50 ítems que se contestan 


\begin{tabular}{|lcccc|}
\hline \multicolumn{5}{c}{ Tabla 1. Estadísticos } \\
& $\mathrm{x}$ & Sx & P.Min & P.Max \\
\hline BS & 20,99 & 8,17 & 1 & 44 \\
\hline Autoconcepto & 70,77 & 8,09 & 41 & 90 \\
\hline Académico & 25,29 & 3,92 & 13 & 33 \\
\hline Social & 13,05 & 2,11 & 5 & 16 \\
\hline Emocional & 17,08 & 3,36 & 9 & 25 \\
\hline Familiar & 15,11 & 2,57 & 6 & 20 \\
\hline Asertividad & 133,1 & 15,36 & 74 & 166 \\
\hline
\end{tabular}

señalando verdadero o falso, pudiendo oscilar la puntuación del sujeto entre 0 y 50 puntos.

El autoconcepto se evaluó mediante el Autoconcepto Forma-A (AFA) (Musitu, García y Gutiérrez, 1991). Consta de 36 ítems que se contestan señalando siempre, algunas veces o nunca, y mide los siguientes factores: académico, social, emocional, familiar y una puntuación total.

La asertividad se midió con el Inventario de Asertividad (Assertion Inventory, Gambrill y Richey, 1975), una vez adaptado el contenido de los ítems a la edad de los sujetos de la muestra. Está formado por 40 ítems, para cada uno de los cuales el sujeto tiene que dar dos respuestas: en primer lugar el grado de incomodidad que le causaría la conducta descrita en el ítem sobre una escala de 1 a 5 puntos; en segundo lugar la probabilidad de realizar la conducta descrita si se presentara la situación en ese momento, puntuando cada ítem desde 1 hasta 5 puntos. En el presente estudio únicamente se recogió la puntuación de probabilidad, la cual puede oscilar entre 40 y 200 puntos.

\subsection{Descripción de la muestra}

Está formada por 243 sujetos de primer curso de Educación Secundaria Obligatoria pertenecientes a 4 colegios privados de la ciudad de Toledo, los cuales fueron seleccionados para participar en un estudio más amplio destinado a la evaluación de un programa de prevención escolar. El $45,7 \%$ de los sujetos son hombres y el $54,3 \%$ mujeres, siendo la media de edad 11,89 años.

\section{RESULTADOS}

\subsection{Consumo de drogas legales}

Los datos de consumo de tabaco obtenidos indican que se hallan en niveles relativamente bajos, encontrándose que un 31,3\% ha fumado alguna vez
BS: Búsqueda de sensaciones Autoconcepto: Autoconcepto general Académico: Autoconcepto académico Social: Autoconcepto social Emocional: Autoconcepto emocional Familiar: Autoconcepto familiar Asertividad: Asertividad $\mathrm{x}$ : Media

Sx: Desviación típica

P.Min: Puntuación mínima P.Máx: Puntuación máxima

tabaco y el 9,9\% lo ha hecho en los últimos 30 días. La edad media a la que se fumó por primera vez es 9,94 años y el consumo medio entre los fumadores es 14,62 cigarrillos en los últimos 30 días y 7,12 cigarrillos en la semana pasada. El consumo es mayor durante el fin de semana (media 4,62 cigarrillos) que de lunes a jueves (media 2,58 cigarrillos).

El consumo de alcohol alcanza niveles más elevados observándose que el $53,5 \%$ de los sujetos ha bebido alcohol alguna vez y un $17,7 \%$ lo ha hecho en los últimos 30 días. La edad media a la que se bebió por primera vez es 10,06 años.

Para analizar los datos sobre la cantidad de alcohol ingerido se utilizó el criterio fijado por la Dirección General de Salud Pública (Ministerio de Sanidad y Consumo) que utiliza el índice UBE (Unidades Básicas Estándar) (Observatorio Español sobre Drogas, 1999).

Entre los sujetos que beben, el consumo medio durante los últimos 30 días es de 11,11 UBE. Por tipos de bebida, el consumo medio de cervezas entre estos sujetos durante los últimos 30 días es de 2,30 UBE y en la semana pasada es de 0,62 UBE, encontrándose un consumo mayor durante el fin de semana (media 0,46 UBE) que de lunes a jueves (media 0,18 UBE). En el caso del vino, el consumo medio en los últimos 30 días es de 3,23 UBE y en la semana pasada es de 1,39 UBE, siendo también el consumo mayor durante el fin de semana (media 0,97 UBE) que de lunes a jueves (media 0,41 UBE). Por último, en cuanto a los licores, el consumo medio en los últimos 30 días es de 5,76 UBE y en la semana pasada es de 3,16 UBE, siendo siempre mayor el consumo durante el fin de semana (media 2,37 UBE) que de lunes a jueves (media 0,79 UBE).

Estos datos reflejan niveles y patrones de consumo de drogas legales similares a los encontrados por otros autores en población escolar (Observatorio Español sobre Drogas, 2000; Sáiz, González, Jiménez et al. 1999; Alonso y del Barrio, 1996b; Espada, Méndez e Hidalgo, 2000). Sin embargo la edad media en la 
que se inició el consumo es menor en los sujetos de la muestra que en los estudios citados.

\subsection{Consumo de cannabis}

El consumo de cannabis está también en niveles relativamente bajos. Así, un 3,7\% ha fumado cannabis alguna vez y la edad media a la que se fumó por primera vez es 12,11 años. En cuanto al consumo de cannabis en los últimos 30 días sólo un 1,6\% lo ha hecho, siendo el consumo medio de cannabis en ese período de 4,75 cigarrillos ("porros"), cantidad que coincide con el consumo en la última semana. Como se esperaba, el consumo es mayor durante el fin de semana (media 3,75 "porros") que de lunes a jueves (media 1 "porro").

Estos datos reflejan consumos más bajos que los encontrados en población escolar española (Observatorio Español sobre Drogas, 2000; González, Sáiz, Quirós et al. 2000), si bien la edad de los sujetos de esta muestra es menor.

Lo que sí resulta preocupante es la temprana edad de inicio al consumo de esta sustancia (12,11 años), ya que una proporción importante de las personas que prueban alguna vez el cannabis continúa después con el consumo (Muñoz, Cruzado y Graña, 1999). Además las edades del primer consumo encontradas son menores a las encontradas en otros trabajos (Sáiz, González, Jiménez et al., 1999; Muñoz, Cruzado y Graña, 1999).

\subsection{Búsqueda de sensaciones y consumo de drogas}

Los datos obtenidos en búsqueda de sensaciones (cuyos estadísticos se incluyen en la tabla 1) presentan relaciones significativas con algunas variables asociadas al consumo de tabaco (ver tabla 2).

Concretamente, los sujetos con mayores puntuaciones en búsqueda de sensaciones es menos probable que rechacen una oferta de tabaco, son los que menos conocimientos tienen sobre las drogas en general y sobre el tabaco en particular, tienen actitudes menos negativas hacia el tabaco, en mayor proporción han visto al mejor amigo/a fumar en los últimos 30 días, perciben menos rechazo al tabaco en sus padres y en su mejor amigo/a, es más probable que hayan fumado alguna vez y que lo hayan hecho en los últimos 30 días, es más probable que no rechacen el consumo de tabaco en su mejor amigo/a, es más probable que hayan recibido ofertas de tabaco, que hayan fumado en presencia de los padres, que consideren que es fácil conseguir tabaco, que tengan intención de fumar en el futuro y que tengan menos expectativas negativas y más positivas respecto al tabaco.
En cuanto al alcohol las relaciones significativas que se han encontrado (ver tabla 3) indican que los sujetos con puntuaciones altas en búsqueda de sensaciones es menos probable que rechacen ofertas de alcohol, tienen actitudes menos negativas hacia esta sustancia, perciben mayor prevalencia de consumo, es más probable que hayan visto a su mejor amigo/a beber en los últimos 30 días, perciben menos rechazo al consumo de esta sustancia en su padre y en su mejor amigo/a, es más probable que hayan bebido alguna vez en su vida y en los últimos 30 días, han bebido más cerveza, vino y licores en los últimos 30 días y en la última semana, se han emborrachado más veces a lo largo de su vida y en los últimos 30 días, es menos probable que rechacen el consumo de alcohol en su mejor amigo/a, reciben más ofertas de alcohol incluso de los padres, es más probable que hayan bebido alcohol en presencia de los padres, perciben menos dificultades para comprar alcohol, es más probable que tengan intención de beber en el futuro y presentan menos expectativas negativas y más expectativas positivas respecto al consumo de esta sustancia.

Por último, por lo que respecta al consumo de cannabis (ver tabla 4), se observa que los sujetos con mayores puntuaciones en búsqueda de sensaciones, es menos probable que rechacen ofertas de cannabis, presentan actitudes menos negativas hacia esta sustancia, es más probable que hayan visto a su mejor amigo/a consumirlo en los últimos 30 días, perciben menos rechazo a esta droga en su mejor amigo/a, es más probable que lo hayan fumado alguna vez, en los últimos 30 días y en la última semana, tienen mejor imagen de los consumidores de cannabis, es menos probable que rechacen el consumo de cannabis por parte de su mejor amigo/a, es más probable que hayan recibido ofertas de cannabis, que les parezca fácil conseguirlo, que tengan intención de fumarlo en el futuro y que tengan menores expectativas negativas y mayores expectativas positivas respecto a su consumo.

\subsection{Autoconcepto y consumo de drogas}

Las puntuaciones obtenidas en relación al autoconcepto general, académico, social, emocional y familiar (ver tabla 1) han mostrado también importantes relaciones con el consumo de drogas (ver tabla 2).

El nivel de autoconcepto general de los sujetos mostró numerosas relaciones significativas con el consumo de tabaco, de manera que los sujetos con mayores puntuaciones en autoconcepto es más probable que rechacen ofertas de tabaco, presentan actitudes más negativas hacia esta sustancia, perciben menores consumos entre los chicos/as de su edad, el consumo materno y de su mejor amigo es menor, es menos probable que hayan fumado en los últimos 30 
días, han fumado menos cigarrillos, han tenido menos ofertas de tabaco y presentan expectativas más negativas con respecto al tabaco.

En cuanto al alcohol, es más probable que los sujetos con puntuaciones altas en esta variable rechacen ofertas de esta sustancia y que nunca hayan bebido alcohol.

Por lo que se refiere al consumo de cannabis no se encontró ninguna relación significativa entre dicho consumo y el autoconcepto general.
Analizando ahora los diferentes tipos de autoconcepto evaluados, el nivel de autoconcepto académico ha mostrado amplias relaciones con el consumo de tabaco (ver tabla 2). Así, los sujetos con puntuaciones altas en dicho factor es más probable que rechacen ofertas de tabaco, tienen actitudes más negativas hacia esta sustancia, perciben menor prevalencia de consumo, presentan menor consumo materno, es menos probable que hayan visto fumar a su mejor amigo/a, perciben más rechazo a que fumen en su

Tabla 2. Relaciones de variables de personalidad con las variables asociadas al consumo de tabaco

\begin{tabular}{|c|c|c|c|c|c|c|c|}
\hline & Bs & Autocon & Academi & Social & Emocion & Familia & Asertiv \\
\hline Tabacno & $* *$ & * & ** & & & ** & ** \\
\hline Conocita & $* *$ & & & & & ** & \\
\hline Totalco & $* *$ & & & & & & \\
\hline Acttaba & $* *$ & * & $* *$ & & & $* *$ & \\
\hline Prevata & & * & * & & & * & \\
\hline Familiata & & * & * & & & & \\
\hline Amigota & $*$ & * & * & * & & * & * \\
\hline Rechapa & * & & & & & & \\
\hline Rechama & * & & & & & & \\
\hline Rechamig & * & & * & & * & * & \\
\hline Consuta & * & & * & & * & * & \\
\hline Con30ta & $*$ & * & * & & & * & \\
\hline Cigar30 & $* *$ & * & $* *$ & & & * & \\
\hline Sematab & & & * & & & & \\
\hline Findeta & & & $* *$ & & & & \\
\hline Imagenfu & & & * & & & & \\
\hline Reaccta & * & & * & & * & & \\
\hline Ofrecta & * & * & * & * & & * & \\
\hline \multicolumn{8}{|l|}{ Ofertafa } \\
\hline Preseta & * & & & & * & & \\
\hline Dificta & * & & * & & & * & \\
\hline Futurta & * & & * & & & * & \\
\hline Expnegt & $* *$ & * & $* *$ & & & & \\
\hline Exppost & * & & & & & & \\
\hline
\end{tabular}

(*) $\mathrm{P}<0.05 ; \quad\left({ }^{*}\right) \mathrm{P}<0.01$

Tabacno: Probabilidad de rechazo de ofertas de tabaco Conocita: Conocimientos acerca del tabaco

Totalco: Conocimientos totales sobre las drogas

Acttaba: Actitudes hacia el tabaco

Prevata: Prevalencia percibida de consumo de tabaco

Familiata: Consumo familiar de tabaco

Amigota: Consumo del mejor amigo/a (últimos 30 días)

Rechapa: Rechazo a que fume percibido en el padre

Rechama: Rechazo a que fume percibido en la madre

Rechamig: Rechazo a que fume percibido en el mejor amigo/a

Consuta: Consumo de tabaco en vida

Con30ta: Consumo de tabaco (últimos 30 días)

Cigar30: Cigarrillos fumados (últimos 30 días)

Sematab: Cigarrillos fumados (lunes a jueves)

Findeta: Cigarrillos fumados (fin de semana)

Imagenfu: Imagen del fumador de tabaco

Reaccta: Reacción al consumo de tabaco por el mejor amigo/a

Ofrecta: Oferta de tabaco recibida del mejor amigo/a

Ofertafa: Oferta de tabaco recibida por la familia

Preseta: Consumo de tabaco en presencia de los padres

Dificta: Dificultad percibida para obtener tabaco

Futurta: Intención de consumir tabaco en el futuro

Expnegt: Expectativas negativas respecto a fumar tabaco

Exppost: Expectativas positivas respecto a fumar tabaco
Bs: Búsqueda de sensaciones

Autocon: Autoconcepto general

Academi: Autoconcepto académico

Social: Autoconcepto social

Emocion: Autoconcepto emocional

Familia: Autoconcepto familiar

Asertiv: Asertividad 
mejor amigo/a, es menos probable que hayan fumado alguna vez o en los últimos 30 días, su consumo de cigarrillos es menor, tienen peor imagen del consumidor de tabaco, es más probable que rechacen el consumo de tabaco por parte de su mejor amigo/a, han recibido menos ofertas de tabaco, perciben mayores dificultades para comprar tabaco, es menos probable que tengan intención de fumar en el futuro y tienen expectativas más negativas respecto al tabaco.

En cuanto al consumo de alcohol (ver tabla 3), los sujetos que puntúan más alto en autoconcepto académico es más probable que rechacen ofertas para beber, tienen actitudes negativas más altas, perciben menor prevalencia de consumo, perciben más rechazo a esta droga en su mejor amigo/a y en su madre, es menos probable que hayan consumido alcohol alguna vez o en los últimos 30 días, es menos probable que beban durante el fin de semana o que se hayan emborrachado, es más probable que rechacen el consumo en su mejor amigo/a, que no hayan recibido ofertas de alcohol, que no hayan recibido oferta de alcohol por parte de los padres o que lo hayan consumido en su presencia, que consideren difícil conseguir alcohol, que no tengan intención de beber en el futuro y que tengan mayores expectativas negativas.

Por lo que se refiere al consumo de cannabis (ver tabla 4), los sujetos con mayores puntuaciones en autoconcepto académico es más probable que rechacen ofertas de esta sustancia o que tengan actitudes más negativas hacia ella. También es menos probable que lo hayan fumado alguna vez, en los últimos 30 días o en la semana pasada, tienen peor imagen de los consumidores de esta droga, es más probable que rechacen el consumo de cannabis por parte de su mejor amigo/a, y tienen mayores expectativas negativas respecto al cannabis.

El nivel de autoconcepto social de los sujetos mostró menos relaciones significativas con el consumo de drogas. En relación al tabaco sólo se observa que los sujetos con mayores puntuaciones en autoconcepto social es menos probable que hayan visto a su mejor amigo/a fumar en los últimos 30 días o que les hayan ofrecido esta sustancia. Por lo que respecta al consumo de alcohol, sólo se ha encontrado que los sujetos con altas puntuaciones nunca han recibido por parte de los padres una oferta y nunca han consumido en presencia de éstos. En cuanto al consumo de cannabis, no apareció ninguna relación significativa.

Más importantes fueron las relaciones encontradas entre el nivel de autoconcepto emocional y las variables asociadas al consumo de drogas. En este sentido se encontró que los sujetos con mayores puntuaciones en este factor son los que perciben menos rechazo a esta droga en su mejor amigo/a, han fumado alguna vez, es más probable que no rechacen el consumo en su mejor amigo/a y han fumado en presencia de los padres (ver tabla 2).

En cuanto al consumo de alcohol, los sujetos con mayores puntuaciones en autoconcepto emocional tienen actitudes menos negativas hacia esta sustancia, es más probable que hayan visto consumir alcohol al mejor amigo/a en los últimos 30 días, perciben menos rechazo a esta droga en su mejor amigo/a, es más probable que hayan bebido en los últimos 30 días, que hayan bebido licores o cervezas en la semana pasada, que hayan bebido vino durante el fin de semana, que no rechacen el consumo en su mejor amigo/a, que reciban ofertas de alcohol por parte de sus padres o que encuentren fácil comprar alcohol (ver tabla 3).

Por último, en relación al cannabis, los sujetos con puntuaciones altas en autoestima emocional es menos probable que rechacen ofertas de esta sustancia, tienen actitudes menos negativas hacia ella, perciben menos rechazo a esta droga en su mejor amigo/a, es más probable que hayan fumado cannabis alguna vez, presentan una imagen positiva hacia los consumidores de dicha sustancia, no rechazan el consumo en su mejor amigo/a, han recibido ofertas de cannabis alguna vez y piensan fumarlo en el futuro (ver tabla 4).

El nivel de autoconcepto familiar de los sujetos mostró también importantes relaciones significativas con el consumo de tabaco (ver tabla 2), de manera que los sujetos con mayores puntuaciones en este factor es más probable que rechacen ofertas de tabaco, tienen actitudes más negativas hacia esta sustancia, perciben menos prevalencia de consumo, perciben más rechazo a esta droga en su mejor amigo/a, es menos probable que hayan fumado en alguna ocasión o en los últimos 30 días, fuman menos, han recibido menos ofertas de tabaco, consideran que conseguir esta sustancia es difícil y no piensan fumar en el futuro.

También se encontró una amplia relación del nivel de autoconcepto familiar con las variables asociadas al consumo de alcohol. Los sujetos con mayores puntuaciones en este factor es más probable que rechacen ofertas de alcohol o que tengan actitudes más negativas hacia él. También es menos probable que hayan bebido alguna vez o en los últimos 30 días, que hayan consumido cervezas, vino o licores en los últimos 30 días o que se hayan emborrachado en ese período. Reaccionarían peor ante un consumo de alcohol por parte de su mejor amigo/a, es menos probable que hayan recibido ofertas de alcohol, incluso por parte de los padres, creen que es difícil comprarlo, no piensan consumirlo en el futuro y tienen mayores expectativas negativas respecto a su consumo (ver tabla 3).

En cuanto al consumo de cannabis, se ha encontrado que los sujetos que puntúan alto en autocon- 
cepto familiar, son los que más probablemente rechazarán ofertas de esta sustancia, tienen actitudes más negativas, es menos probable que hayan consumido esta sustancia alguna vez, en los últimos 30 días o durante la semana pasada y reaccionarían mal ante el consumo de su mejor amigo/a. También es menos probable que hayan recibido ofertas de cannabis o que piensen fumarlo en el futuro (ver tabla 4).

Tabla 3. Relaciones de variables de personalidad con las variables asociadas al consumo de alcohol

\begin{tabular}{|c|c|c|c|c|c|c|c|}
\hline & Bs & Autocon & Academi & Social & Emocion & Familia & Asertiv \\
\hline Alcohno & $* *$ & $*$ & * & & & ** & \\
\hline \multicolumn{8}{|l|}{ Conocial } \\
\hline \multicolumn{8}{|l|}{ Totalco } \\
\hline Actalco & $* *$ & & ** & & $* *$ & $* *$ & \\
\hline Prevaal & $*$ & & * & & & & \\
\hline \multicolumn{8}{|l|}{ Familiaal } \\
\hline Amigoal & * & & & & * & & \\
\hline Rechapa & * & & & & & & \\
\hline Rechama & & & * & & & & \\
\hline Rechamig & * & & * & & * & & \\
\hline Consual & * & * & * & & & * & \\
\hline Con30al & * & & * & & * & * & \\
\hline Cerve30 & $* *$ & & ** & & & ** & \\
\hline Vino30 & ** & & $* *$ & & & ** & \\
\hline Copas30 & $* *$ & & $* *$ & & * & $* *$ & \\
\hline Semacer & $*$ & & & & & & \\
\hline Findece & $* *$ & & $* *$ & & & ** & \\
\hline \multicolumn{8}{|l|}{ Semavin } \\
\hline Findevi & $* *$ & & $* *$ & & * & $* *$ & \\
\hline Semacop & $* *$ & & & & ** & * & * \\
\hline Findeco & $* *$ & & * & & & ** & \\
\hline Borrach & $* *$ & & * & & & $* *$ & \\
\hline Borra30 & ** & & & & & * & \\
\hline \multicolumn{8}{|l|}{ Imagenal } \\
\hline Reaccal & * & & $*$ & & * & * & \\
\hline Ofrecal & * & & * & & & * & \\
\hline Ofertafa & $*$ & & * & * & * & * & \\
\hline Preseal & * & & * & * & & & \\
\hline Difical & * & & * & & * & * & \\
\hline Futural & $*$ & & * & & & * & \\
\hline Expnegt & $* *$ & & ** & & & * & \\
\hline Exppost & $* *$ & & & & & & \\
\hline
\end{tabular}

\section{(*) $P \leq 0.05 ;\left(^{* *}\right) P \leq 0.01$}

Alcohno: Probabilidad de rechazo de oferta de alcohol Conocial: Conocimientos acerca del alcohol

Totalco: Conocimientos totales sobre las drogas Actalco: Actitudes hacia el alcohol

Prevaal: Prevalencia percibida de consumo de alcohol Familiaal: Consumo familiar de alcohol

Amigoal: Consumo del mejor amigo/a (últimos 30 días) Rechapa: Rechazo a que beba percibido en el padre

Rechama: Rechazo a que beba percibido en la madre

Rechamig: Rechazo a que beba percibido en mejor amigo/a

Borra30: № episodios de embriaguez (últimos 30 días)

Findeco: Copas bebidas (fin de semana)

Consual: Consumo de alcohol en vida

Con30al: Consumo de alcohol (últimos 30 días)

Cerve30: Cervezas bebidas (últimos 30 días)

Vino30: Vasos de vino bebidos (últimos 30 días)

Copas30: Copas bebidas (últimos 30 días)

Semacer: Cervezas bebidas (lunes a jueves)

Findece: Cervezas bebidas (fin de semana)

Semavin: Vasos de vino bebidos (lunes a jueves)

Findevi: Vasos de vino bebidos (fin de semana)

Semacop: Copas bebidas (lunes a jueves)
Borrach: $N^{\circ}$ total de veces que se ha embriagado Imagenal: Imagen de bebedor de alcohol

Reaccal: Reacción al consumo por mejor amigo/a Ofrecal: Oferta de alcohol recibida de mejor amigo Padreal: Oferta de alcohol recibida de la familia

Preseal: Consumo alcohol en presencia de padres

Difical: Dificultad percibida para obtener alcohol

Futural: Intención de consumir alcohol en el futuro

Expnegt: Expectativas negativas respecto a alcohol

Exppost: Expectativas positivas respecto a alcohol

Bs: Búsqueda de sensaciones

Autocon: Autoconcepto general

Academi: Autoconcepto académico

Social: Autonconcepto social

Emocion: Autoconcepto emocional

Familia: Autoconcepto familiar

Asertiv: Asertividad 


\subsection{Asertividad y consumo de drogas}

Por último, las puntuaciones en probabilidad de conducta asertiva (ver tabla 1) muestran escasas relaciones con el consumo de drogas, independientemente de la sustancia considerada (ver tablas 2, 3 y 4).

Así, con respecto al tabaco los sujetos con puntuaciones altas en asertividad es más probable que rechacen ofertas para fumar y es menos probable que hayan visto fumar a su mejor amigo/a.

En cuanto al consumo de alcohol, curiosamente se ha encontrado que las puntuaciones en la escala de probabilidad muestran una correlación positiva significativa con el consumo de lunes a jueves de licores $(r=0,13, p=0,043)$.

No se han encontrado ninguna relación significativa en lo concerniente al consumo de cannabis.

5 Por el contrario, sí se han encontrado múltiples relaciones significativas entre la asertividad relacionada con las sustancias (probabilidad de decir no a una oferta de tabaco, alcohol o cannabis) y el consumo de éstas, de manera que los sujetos más asertivos son los que menos las consumen.

\section{DISCUSIÓN}

Al igual que se ha encontrado en otros estudios (Mendoza, 1987; Alonso y del Barrio, 1996b; Sáiz , González, Jiménez et al., 1999; Observatorio Español sobre Drogas, 1999; 2000) el tabaco y el alcohol son las dos sustancias psicoactivas que más consumen los escolares españoles, lo cual refleja una cierta permisividad y facilidad de obtención, debido a ser éstas sustancias muy integradas social y culturalmente en nuestro país.

Igualmente se observa la existencia de un importante consumo de cannabis, confirmándose que, después del alcohol y el tabaco, es la sustancia psicoactiva más extendida entre los adolescentes y la que ha experimentado un mayor incremento entre los jóvenes (Sáiz, González, Jiménez et al., 1999; Graña y Muñoz-Rivas, 2000).

Del mismo modo, los datos avalan una vez más la existencia de un patrón de consumo que se caracteriza por llevarse a cabo principalmente durante los fines de semana y por centrarse en el consumo de licores y combinados, tal como ha sido ya ampliamente descrito por otros autores (Alonso y del Barrio, 1996b; Espada, Méndez e Hidalgo, 2000; Observatorio Español sobre Drogas, 1999; 2000).

Como se preveía, se han encontrado numerosas relaciones significativas entre las variables de personalidad analizadas y el consumo de drogas, así como con el resto de las variables asociadas a dicho consumo. Por ejemplo, niveles altos de búsqueda de sensaciones presentan una intensa relación con muchas de las variables estudiadas, confirmándose la relación de este factor con el consumo de drogas legales e ilegales, puesta ya de manifiesto por otros muchos trabajos (Luengo, Otero-López, Romero et al., 1996; Sáiz, González, Paredes et al., 2001; Sáiz, González, Jiménez et al. 1999; González, Sáiz, Quirós et al., 2000; Calafat, Juan, Becoña et al., 2000).

Al respecto, los datos apoyan los resultados aportados por Luengo, Otero-López, Romero et al. (1996) quienes señalan que la búsqueda de sensaciones es una variable relevante para diferenciar a los adolescentes no consumidores de aquellos que comienzan a consumir. El presente trabajo permite confirmar el valor predictivo de la búsqueda de sensaciones en relación, no sólo al consumo habitual, sino también al consumo experimental, ya que tanto los sujetos que han consumido experimentalmente, como los que consumen habitualmente tabaco, alcohol y cannabis, puntúan más alto en búsqueda de sensaciones que los que nunca han consumido esas sustancias.

En consecuencia parece que la variable búsqueda de sensaciones juega un papel importante para la identificación de situaciones de riesgo, así como para la discriminación de diferentes patrones de consumo.

En cuanto al autoconcepto los resultados son más variados. Por un lado, indican que puntuaciones altas en los factores académico y familiar del autoconcepto parecen tener un efecto protector respecto al consumo de drogas legales e ilegales, ya que se relacionan con menores consumos de tabaco, alcohol y cannabis, así como con resultados menos vinculados al consumo de drogas en el resto de las variables estudiadas.

Efectivamente, los datos indican que tener un alto autoconcepto familiar puede ser una variable protectora con respecto al consumo de tabaco, alcohol y cannabis, lo cual es coherente con los resultados citados por otros autores que sugieren que el apoyo familiar, la calidad de la relación padres-hijos, el afecto familiar... actúan como factores de protección, incluso amortiguando la influencia del grupo de iguales en el consumo de drogas (Frauenglass, Routh, Pantin et al., 1997; Recio, Santos, Sánchez et al., 1991; Farrell y White, 1998).

De acuerdo con lo sugerido por Romero, Luengo y Otero-López (1995), la explicación de estos resultados puede estar en que una baja autoestima en la familia y en la escuela puede actuar como motivadora de la iniciación o el progreso en el consumo de drogas debido a que una baja autovaloración en estos ámbitos puede debilitar la vinculación del individuo con los grupos convencionales impulsándole a buscar grupos de refe- 
Tabla 4. Relaciones de variables de personalidad con las variables asociadas al consumo de cannbis

\begin{tabular}{|c|c|c|c|c|c|c|c|}
\hline & Bs & Autocon & Academi & Social & Emocion & Familia & Asertiv \\
\hline Cannabno & $* *$ & & * & & ** & * & \\
\hline \multicolumn{8}{|l|}{ Conocican } \\
\hline \multicolumn{8}{|l|}{ Totalco } \\
\hline Actcann & $* *$ & & $*$ & & $* *$ & $* *$ & \\
\hline \multicolumn{8}{|l|}{ Prevacan } \\
\hline \multicolumn{8}{|l|}{ Familiacan } \\
\hline Amigocan & * & & & & & & \\
\hline \multicolumn{8}{|l|}{ Rechapa } \\
\hline \multicolumn{8}{|l|}{ Rechama } \\
\hline Rechamig & * & & & & * & & \\
\hline Consucan & * & & * & & * & * & \\
\hline Con30can & * & & & & & * & \\
\hline Cannab30 & $* *$ & & ** & & & ** & \\
\hline Semacann & $* *$ & & $* *$ & & & * & \\
\hline Findecan & $* *$ & & $* *$ & & & $* *$ & \\
\hline Imagenfu & $*$ & & * & & * & & \\
\hline Reacccan & * & & * & & * & * & \\
\hline Ofreccan & * & & & & * & * & \\
\hline \multicolumn{8}{|l|}{ Ofertafa } \\
\hline \multicolumn{8}{|l|}{ Presecan } \\
\hline Dificcan & $*$ & & & & & & \\
\hline Futurcan & $*$ & & & & * & $*$ & \\
\hline Expnegt & $* *$ & & ** & & & & \\
\hline Exppost & $* *$ & & & & & & \\
\hline
\end{tabular}

$(*) P \leq 0.05 ;\left(^{*}\right) P \leq 0.01$

Cannabno: Probabilidad de rechazo de ofertas de cannabis Conocica: Conocimientos acerca del cannabis

Totalco: Conocimientos totales sobre las drogas

Actcann: Actitudes hacia el cannabis

Prevacan: Prevalencia percibida de consumo de cannabis

Familiata: Consumo familiar de cannabis

Amigocan: Consumo del mejor amigo/a (últimos 30 días)

Rechapa: Rechazo a que fume percibido en el padre

Rechama: Rechazo a que fume percibido en la madre

Rechamig: Rechazo a que fume percibido en el mejor amigo/a

Consucan: Consumo de cannabis en vida

Con30can: Consumo de cannabis (últimos 30 días)

Cannab30: Cigarrillos de cannabis fumados (últimos 30 días)

Semacann: Cigarrillos de cannabis fumados (lunes a jueves)

Findeca: Cigarrillos de cannabis fumados (fin de semana)

Imagenfu: Imagen del fumador de cannabis

Reacccan: Reacción al consumo de tabaco por el mejor amigo/a

Ofreccan: Oferta de cannabis recibida por el mejor amigo/a

Ofertafa: Oferta de cannabis recibida por la familia

Presecana: Consumo de cannabis en presencia de los padres

Dificcan: Dificultad percibida para obtener cannabis

Futurta: Intención de consumir cannabis en el futuro

Expnegt: Expectativas negativas respecto a fumar cannabis

Exppost: Expectativas positivas respecto a fumar cannabis
Bs: Búsqueda de sensaciones Autocon: Autoconcepto general

Academi: Autoconcepto académico

Social: Autoconcepto social

Emocion: Autoconcepto emocional

Familia: Autoconcepto familiar

Asertiv: Asertividad rencia alternativos y nuevas vías de acción a través de las que poder reponer la autoestima.

Por el contrario, los resultados obtenidos en relación al autoconcepto general muestran que esta variable tiene una débil relación con el consumo de alcohol y ninguna relación con el consumo de cannabis. Unicamente con el consumo de tabaco presenta relaciones importantes.

Aunque ello contradice la relación entre baja autoestima y consumo de drogas señalada por distintos trabajos (Jackson, 1997), también es cierto que la ambigüedad de la relación entre el autoconcepto y el consumo de estas sustancias ya ha sido sugerida por Graña y Muñoz-Rivas (2000) quienes concluyen que el autoconcepto-autoestima puede aparecer como una variable predictora tanto de un mayor como de un menor riesgo de consumo de sustancias legales e ilegales. Así, una mayor satisfacción personal es un adecuado predictor de una menor implicación del adolescente en el consumo de drogas, pero también 
podría colocarle en una situación de mayor seguridad con respecto al uso de drogas viéndose más capacitado para, bien poder iniciar el consumo y experimentar con el alcohol y el tabaco o bien, avanzar en el consumo de otras drogas.

De acuerdo con esta hipótesis se analizó la existencia de una posible interacción entre las expectativas negativas hacia las diferentes drogas y el autoconcepto. Sin embargo los datos no permiten apoyar la citada explicación ya que no se encontró una relación diferente entre expectativas negativas y consumo de drogas en función del nivel de autoconcepto de los sujetos.

También ha sido ampliamente debatida la relación entre el fracaso escolar y el consumo de drogas. Efectivamente, si bien la mayoría de los estudios ha puesto de manifiesto que el fracaso escolar es un factor de riesgo en relación al consumo de drogas (Alonso y del Barrio, 1996b; Hu, Lin y Kuler, 1998), también es cierto que algunos autores (Evans y Skager, 1992) han encontrado consumidores de drogas en todos los niveles de ejecución escolar, lo cual pone en duda el valor predictivo de esta variable. Igualmente, en población juvenil española, Calafat, Juan, Becoña et al (2000) subrayan la necesidad de ser prudentes al relacionar fracaso escolar y consumo de drogas.

Los datos obtenidos en relación al fracaso escolar, al autoconcepto académico y a su relación con el consumo de drogas, permiten avanzar en la polémica planteada entorno a estas variables.

Por un lado, los resultados indican que el fracaso escolar (valorado a partir del número de suspensos en el curso anterior) no presenta ninguna relación con el consumo de drogas, por lo que debe cuestionarse su valor como factor de riesgo. Al mismo tiempo, el autoconcepto académico sí presenta una intensa relación con el consumo de drogas y no se relaciona con el fracaso escolar, lo cual indica que se elabora en base a otros muchos elementos independientes de las calificaciones (opinión percibida en los profesores, niveles de exigencia de los padres, percepción de su esfuerzo, autovaloración de los resultados, valoración de su propio comportamiento en clase, etc).

Todo ello indica que una deficiente ejecución de las tareas escolares puede ser, efectivamente, una fuente de riesgo, pero no tanto referida al rendimiento objetivo del alumno -que se traduce en sus calificaciones- como a la percepción que el sujeto tiene de su trabajo, de su esfuerzo, de su comportamiento, de las opiniones de los demás, etc, todo lo cual se refleja en el autoconcepto académico.

Por todo ello, la polémica planteada en torno al fracaso escolar, que ha llevado a cuestionar su valor como factor de riesgo y, consecuentemente, a quitar importancia a la mejora del trabajo escolar como área de intervención preventiva, puede resolverse sustitu- yendo el interés en reducir el fracaso escolar (entendido como déficit objetivo en la ejecución) por un trabajo más amplio y centrado en el desarrollo del autoconcepto académico, lo cual implicaría no tanto la mejora de los resultados objetivos como la adecuación de los niveles de exigencia, el refuerzo del logro, el reconocimiento del esfuerzo realizado, la autovaloración y el autocontrol del comportamiento, la expresión de valoraciones positivas en padres y profesores, etc.

Sería de gran utilidad que investigaciones posteriores profundizaran en la relación existente entre autoconcepto académico y consumo de drogas ya que el rendimiento escolar es una variable ampliamente incluida en los estudios de consumo de drogas en población escolar por su supuesto valor predictivo y, de confirmarse la hipótesis planteada, debería ser sustituida por una medida del autoconcepto académico.

Sorprendentes han sido los resultados obtenidos en relación al autoconcepto emocional. En contra de lo esperado, los sujetos que puntúan alto en este factor son los que presentan mayor probabilidad de consumo de drogas legales e ilegales.

Las puntuaciones altas en este factor reflejan un buen control emocional, así como un elevado grado de aceptación de las emociones producidas por su conducta (por ej., se trata de sujetos que raramente "se preocupan mucho por todo", "tienen miedo de algunas cosas" o "se avergüenzan de muchas cosas que hacen"). Quizá por estos motivos se trate de sujetos que no dudan en probar las drogas, aunque no necesariamente se impliquen en un consumo continuado de éstas (por ejemplo, un elevado autoconcepto emocional se relaciona sólo con haber probado el tabaco o el cannabis, pero no con su consumo en los últimos 30 días).

Estos resultados sugieren la necesidad de explorar más cuidadosamente la relación descrita por algunos trabajos entre neuroticismo y consumo de drogas (Sáiz, González, Jiménez et al., 1999) o entre susceptibilidad a la ansiedad y consumo de alcohol (Norton, Rockman, Ediger et al., 1997). Posiblemente, la relación entre el consumo de drogas y este tipo de variables no sea lineal, sino que niveles muy altos o muy bajos de control emocional pueden relacionarse con el consumo de estas sustancias, mientras que en los niveles intermedios se daría la menor probabilidad de consumo.

Una vez más serán necesarias nuevas investigaciones para aclarar el valor de estas variables como factores de riesgo en relación al consumo de drogas y su papel en el inicio y mantenimiento del consumo de drogas legales e ilegales.

En cuanto al autoconcepto social, los resultados muestran que apenas se relaciona con el consumo de drogas. Puesto que este factor del autoconcepto se 
define en base a la capacidad del sujeto para relacionarse con los demás, parece claro que la calidad de las relaciones sociales de los escolares no tienen relación con el consumo de drogas, lo cual contradice las explicaciones de tal consumo que lo interpretan como un recurso para enfrentarse a unas relaciones sociales que se viven de forma problemática.

Ello es absolutamente coherente con los resultados obtenidos en relación a la asertividad, la cual no presenta apenas ninguna relación con el consumo de drogas. Estos resultados se inscriben en la polémica planteada en cuanto a la relación de la asertividad y las habilidades sociales con el consumo de drogas. Por un lado resultan contradictorios con las conclusiones a las que llegan otros autores que apoyan la relación de las habilidades sociales generales con el consumo de drogas, aconsejando su desarrollo como mecanismo de prevención (Hover y Gaffney, 1991). Sin embargo, por otro lado, parecen confirmar la escasa relación entre estas variables encontrada en otros trabajos (Alonso y Del Barrio, 1996a) y apoyan también las conclusiones de Ashby, Baker y Botvin (1989) quienes señalaron que la asertividad general no se relaciona con el consumo de drogas, aunque sí la asertividad relacionada con las sustancias y la asertividad social.

Efectivamente, los resultados obtenidos en el presente trabajo ponen de manifiesto que la calidad de las relaciones sociales de los sujetos o el comportamiento asertivo general no tienen relación con el consumo de drogas legales ni ilegales, mientras que una alta asertividad relacionada con las sustancias sí se asocia a menores consumos. Ello invita a revisar los numerosos programas de prevención que se basan en el desarrollo de la asertividad general, los cuales quizá podrían mejorar su eficiencia -y quizá también su efectividad- centrándose en el desarrollo de la asertividad relacionada con las sustancias.

También hay que tener en cuenta que el presente trabajo tiene importantes limitaciones. Por un lado, se trata de una muestra muy pequeña y en absoluto representativa del grupo de edad al que pertenece. Por otro lado, algunos de los instrumentos -si bien han sido ampliamente utilizados- pueden presentar inconvenientes. Por ejemplo Carrasco, Clemente y Llavona (1989) señalan que el inventario de aserción de Gambrill y Richey no reúne suficientes elementos que permitan evaluar de manera completa la conducta asertiva, porque aunque recoge la mayoría de los comportamientos que se han de considerar a la hora de evaluar la conducta asertiva (conductas de oposición, enfrentamiento y defensa, dar y aceptar cumplidos, hacer peticiones, expresar sentimientos positivos y afecto, expresar el desagrado y expresar limitaciones personales), no hace referencia a la manifestación de opiniones personales, al iniciar y mantener conversaciones, ni a la expresión del enfado.
En cualquier caso, cabe señalar como conclusión la importancia de algunas variables de personalidad que deben ser tenidas en cuenta por los programas dirigidos a prevenir el consumo de drogas en población escolar. Según los resultados obtenidos, estos programas deben facilitar medios adecuados para la búsqueda de sensaciones, por ejemplo a través de actividades de tiempo libre. Igualmente deben promover el ajuste de los niveles de exigencia y el reconocimiento de los logros escolares para mejorar el autoconcepto académico, así como mejorar las relaciones entre padres e hijos para favorecer el autoconcepto familiar.

En sentido contrario, conviene revisar la utilidad de las intervenciones destinadas a la mejora del autoconcepto general o del control emocional. Igualmente es necesario profundizar en la evaluación de los programas centrados en el desarrollo de las relaciones sociales generales, ya que el autoconcepto social y la asertividad general no parecen relacionarse con el consumo de drogas. Por el contrario, la efectividad de los programas podría mejorarse con una mayor atención a la asertividad relacionada con las drogas.

\section{CONCLUSIONES.}

1) La búsqueda de sensaciones presenta una intensa relación con el consumo de drogas y es una variable muy importante a la hora de identificar situaciones de riesgo.

2) El autoconcepto es una variable compleja integrada por muy diversos factores que se relacionan de forma diferente con el consumo de drogas.

3) Un elevado autoconcepto familiar y académico parece ejercer un papel protector respecto al consumo de drogas, por lo que su desarrollo debe ser considerado como un importe objetivo de los programas de prevención. Al respecto, el autoconcepto académico no debe confundirse con el rendimiento escolar, siendo necesario para su desarrollo un trabajo más complejo que la simple mejora de los resultados escolares.

4) El autoconcepto académico presenta una relación mucho más intensa con el consumo de drogas que el rendimiento escolar, por lo que debe sustituir a éste en la identificación de situaciones de riesgo.

5) Tanto el autoconcepto social como la asertividad general no tienen apenas relación con el consumo de drogas, mientras que sí la tiene la asertividad relacionada con las sustancias. Por ello los programas de prevención deberían centrarse en el desarrollo de esta última y no tanto en el trabajo sobre habilidades sociales generales. 
6) Es necesario profundizar en el conocimiento de la relación existente entre el autoconcepto emocional y el consumo de drogas, ya que altas puntuaciones en este factor podrían facilitar dicho consumo.

\section{BIBLIOGRAFÍA}

Alonso, C. y del Barrio, V. (1996a): Autoestima, asertividad, lugar de control y consumo de drogas legales. Revista de Psicología de la Educación, 19, 5-20.

Alonso, C y del Barrio, V. (1996b): Consumo de drogas legales y factores asociados al ambiente escolar. Psicología Educativa, 2 (1), 91-112.

Ashby, T.; Baker, E. y Botvin, G.J. (1989): Dimensions of assertiveness: differential relationships to substance use in early adolescence. Journal of Consulting and Clinical Psychology, 57(4), 473-478.

Calafat, A., Juan, M., Becoña, E., Fernández, C., Gil, E., Palmer, A., Sureda, P. y Torres, M.A. (2000). Salir de marcha y consumo de drogas. Madrid: Delegación del Gobierno para el Plan Nacional sobre Drogas. Ministerio del Interior.

Carrasco, I.; Clemente, M. y Llavona, L. (1989): Análisis del inventario de aserción de Gambrill y Richey. Estudios de Psicología, 37, 63-74.

Espada, J.P.; Méndez, F.X. e Hidalgo, M.D. (2000): Consumo de alcohol en escolares: Descenso de la edad de inicio y cambios en los patrones de ingesta. Adicciones, 12(1), 57-63.

Evans, W.P. y Skager, R. (1992). Academically successful drug users: an oxymoron?. Journal of Drug Education, 22(4), 353-365.

Farrell, A. y White, K. (1998): Peer inluences and drug use among urban adolescents: Family, structure and parent-adolescent relationship as protective factors. Journal of Consulting and Clinical Psychology, 66,(2), 248-258.

Frauenglass, S.; Routh, D.; Pantin, H. y Mason, C. (1997): Family support decreases influence of desviant peers on hispanic adolescents substance use. Journal of Clinical Child Psychology, 26(1), 15-23.

Gambrill, E.D. y Richey, C.A. (1975). An assertion inventory for use in assessment and research. Behavior Therapy, $6,550-561$.

González, M.; Ibáñez, I. y Peñate, W. (1997): Consumo de alcohol, búsqueda de sensaciones y dimensiones básicas de personalidad. Análisis y Modificación de Conducta, 23(89), 385-405.

González, M.P.; Sáiz, P.A.; Quirós, M. y López, J.L. (2000): Personalidad y uso-abuso de cannabis. Adicciones, 12, suplemento 2, 185-196.

Graña, J.L. y Muñoz-Rivas, M. (2000): Factores psicológicos de riesgo y de protección para el consumo de drogas en adolescentes. Psicología Conductual, 8(2), 249269.
Graña, J.L.; Muñoz-Rivas, M.; Andreu, J.M. y Peña, M.E. (2000): Variables psicológicas relacionadas con el consumo de drogas en adolescentes: depresión y autoconcepto. Revista de Drogodependencia, 25(1), 170-181.

Gustafson, R. y Kälmén, H. (1996): Alcohol and the disinhibition of social assertive behaviours. European Addiction Research, 2, 73-77.

Hover, J.T. y Gaffney, L.R. (1991). The relationship between social skills and adolescent drinking. Alcohol and Alcoholism, 26(2), 207-214.

Hu, T.; Lin, Z. y Kuler, T. (1998): Teenage smoking, attempts to quit and school performance. Americam Journal of Public Health, 88(6), 940-943.

Jackson, C. (1997): Initial and eexperimental stages of tobacco and alcohol use during late childhood: relation to peer, parent, ada personal risk factors. Adicctive Behaviours, 22(5), 685-698.

Luengo, M.A.; Otero-López, J.M.; Romero, E. y Gómez-Fraguela, J.A. (1996): Efectos de la necesidad de búsqueda de sensaciones sobre la involucración en el consumo de drogas de los adolescentes. Análisis y Modificación de Conducta, 22(86), 683-709.

Mendoza, R. (1987): Consumo de alcohol y tabaco en los escolares españoles. Comunidad y Drogas, 5/6, 83102.

Muñoz, M.J.; Cruzado, J.A. y Graña, J.L. (1999): Consumo de drogas en adolescentes de la Comunidad de Madrid. Adicciones, 11(4), 311-322.

Musitu, G., García, F. y Gutiérrez, M. (1991). Autoconcepto Forma-A (AFA). Madrid: Tea.

Norton, G.; Rockman, G.; Ediger, J.; Pepe, C.; Goldberg, S.; Cox, B. y Asmundson, G. (1997): Anxiety sensitivity and drug choice in individuals seeking treatment for substance abuse. Behavior Research and Therapy, 35(9), 859-862.

Observatorio Español sobre Drogas (1999): Informe $\mathbf{n}^{0} \mathbf{1}$ Madrid: Ministerio del Interior. Delegación del Gobierno para el Plan Nacional sobre Drogas.

Observatorio Español sobre Drogas (2000): Informe $\mathbf{n}^{\circ} \mathbf{3}$. Madrid: Ministerio del Interior. Delegación del Gobierno para el Plan Nacional sobre Drogas.

Pérez, J., Ortet, G., Plá, S. y Simó, S. (1987). Escala de Búsqueda de Sensaciones para Niños y Adolescentes (EBS-J). Evaluación Psicológica, 3, 283-290.

Recio, J.L.; Santos, C.; Sánchez, M.A.; Escamilla, J.P.; Plaza, L.A. y Barahona, M.J. (1991): El papel de la familia, los compañeros y la escuela en el abuso adolescente de drogas. Madrid: Cruz Roja Española.

Roches, J.E. y Jason, L.A. (1990): A social stress model of substance abuse. Journal of Consulting and Clinical Psychology, 58(4), 395-401.

Romero, E.; Luengo, M.A. y Otero-López, J.M. (1995): La relación entre autoestima y consumo de drogas en los adolescentes: un análisis longitudinal. Revista de Psicología Social, 10(2), 149-159.

Sáiz, P.A.; González, M.P.; Jiménez, L.; Delgado, Y.; Liboreiro, M.J.; Granda, B. y Bobes, J. (1999): Consumo de alco- 
hol, tabaco y otras drogas y rasgos de personalidad en jóvenes de enseñanza secundaria. Adicciones, 11(3), 209-220.

Sáiz, P.A; González, M.P.; Paredes, B.; Delgado, J.M.; López, J.L.; Martínez, S. y Bobes, J. (2001): Consumo de MDMA (éxtasis) en estudiantes de secundaria. Adicciones, 13(2), 159-171.
Zuckerman, M., Eysenck, S.B. y Eysenck, A.J. (1978). Sensation seeking in England and American: cross-cultural age and sex comparisons. Journal of Consulting and Clinical Psychology, 46, 139-149. 
